\title{
Questes
}

\section{Dire par non-dire : quelques remarques sur la critique de particuliers dans les sotties}

\section{Taku Kuroiwa}

\section{(2) OpenEdition}

1 Journals

\section{Édition électronique}

URL : http://journals.openedition.org/questes/2792

DOI : 10.4000/questes. 2792

ISSN : 2109-9472

Éditeur

Les Amis de Questes

\section{Édition imprimée}

Date de publication : 15 juin 2004

Pagination : $36-41$

ISSN : 2102-7188

\section{Référence électronique}

Taku Kuroiwa, « Dire par non-dire : quelques remarques sur la critique de particuliers dans les sotties », Questes [En ligne], 7 | 2004, mis en ligne le 19 mai 2016, consulté le 22 septembre 2020. URL : http:// journals.openedition.org/questes/2792 ; DOI : https://doi.org/10.4000/questes.2792

Ce document a été généré automatiquement le 22 septembre 2020.

(c) Association des amis de «Questes » 


\title{
Dire par non-dire : quelques remarques sur la critique de particuliers dans les sotties
}

\author{
Taku Kuroiwa
}

1 À la fin du XV et au début du XVI e siècle, il apparaît une série d'écrits théoriques sur la poétique vernaculaire, où on trouve aussi la mention de la satire en tant que genre littéraire. Bien évidemment, ces efforts de justification de la satire littéraire n'ont pas facilement débouché sur la liberté critique, à cause des obstacles politiques et moraux. En prenant comme exemple quelques pièces dramatiques relevant de la sottie, genre dramatique où apparaissent les sots qui portent le costume traditionnel du fou de cour, nous voulons aborder quelques problèmes concernant la transmission de discours interdits ou de tabous ${ }^{1}$.

\section{Justification de la littérature satirique et ses difficultés}

2 Lors de la publication des textes d'Horace, de Juvénal et de Perse, les érudits du XVe et du XVI ${ }^{e}$ siècles ont dû défendre la légitimité de ces textes qui paraissaient difficilement acceptables aux autorités tant ecclésiastiques que royales ${ }^{2}$. Josse Bade, imprimeurhumaniste du début du XVI siècle qui a aussi édité et commenté les oeuvres d'Horace, de Perse et de Juvénal, fait remonter l'origine de la satire latine à la comédie ancienne grecque, et il compare la satire latine au sermon dans l'église pour souligner la valeur morale et édifiante de la satire. Voici un passage tiré de sa préface en latin aux comédies de Térence qu'il a publiées lui-même :

Puis, quand on eut interdit de s'attaquer à la réputation de personnes vivantes en les nommant par leur nom dans les comédies ou dans les satires, on voit apparaitre, dans un troisième temps, la comédie nouvelle dont il est question dans cette préface et qui a été inventée par les Grecs. Mais c'est chez les Romains seulement que l'on trouve la nouvelle satire, poème qu'on qualifie, à tort, de censeur de vices, puisque le poète satirique remplit le même rôle que le prédicateur dans l'Eglise 
chrétienne; car même Horace a voulu donner le nom de sermones à ses compositions [unde etiam Horatius satyras suas sermones appellare voluit]. ${ }^{3}$

Par ailleurs, dans quelques écrits sur l'art poétique vernaculaire de l'époque, la critique des particuliers avec la mention de leurs noms propres est vivement déconseillée : en rapprochant la sottie vernaculaire de la satire latine, Jean Bouchet, dans sa célèbre épître adressée aux étudiants de l'Université de Poitiers, précise que la critique des particuliers vient de la « folle affection $»^{4}$.

Et tiercement aultres poetes sont / Satyres dictz, qui tous leurs metres font / Reprehensifz de tous pechez publiques / Les reprenans par leurs vers satyriques, / Qui sont picquans voire iusques au sang, / Ne craignans rien, mais de tout parlent franc / Louans vertuz, et detestans tout vice / Sans espargner par crainte aulcun conuice, / Lesquelz peuent bien ieunes gens eriger / A bonnes meurs, et vertuz eriger, / Et a laisser les mauluaise coustumes / Des vitieux remplies d'apostumes.

Horatius, Perse, et aussi Iuuenal, / Furent aucteurs de ce ieu Satyral / Entre Latins, comme on veoit par leurs liures, / Lesquelz ne sont de sentences deliures.

Mais par autant que de detraction / Vsent souuent par folle affection / Nommans aucuns, et faisans du scandalle / On dit Satyle estre vne chose malle. / En France elle a de sotie le nom,/ Parce que sotz des gens de grand renom / Et des petits jouent les grands follies, / Sur eschaffaux, en parolles polies, / Qui est permis par les princes et Roys / A cette fin qu'ils sçachent les derroys / De leur conseil qu'on ne leur ause dire / Desquelz ils sont advertis par Satyre. / Le roy Loys douziesme desiroit / Qu'on les jouast a Paris, et disoit /Que par tels jeux il sçauoit maintes faultes / Qu'on luy celoit par surprinses trop caultes. ${ }^{5}$

Cependant, comme on peut facilement l'imaginer, cette proscription n'était pas toujours observée par les joueurs et les auteurs de pièces de théâtre, et ils ont été souvent punis par l'autorité royale à cause de leurs critiques contre les notables ${ }^{6}$.

\section{Critique de particuliers dans les sotties}

5 Cependant, si l'on examine les sotties conservées jusqu'à aujourd'hui, on s'aperçoit qu'il $\mathrm{y}$ a assez peu de pièces qui présentent une critique des particuliers (au moins avec leurs noms propres), sinon des mentions qui correspondent bien à la politique royale lors de la représentation ou de la publication ${ }^{7}$. Même dans la Sotye nouvelle des croniqueurs ${ }^{8}$ et la Sottie nouvelle de l'Astrologue ${ }^{9}$, deux sotties qui sont conservées en manuscrit et qui contiennent un assez grand nombre de mentions de particuliers et d'événements historiques, on évite souvent de parler des notables avec leurs noms à l'aide de blasons ou de noms de constellations, même quand ils font l'éloge des personnes en question. Par ailleurs, dans les sotties du Recueil Trepperel, recueil factice de brochures en format agenda imprimées au début du XVIe siècle, on rencontre assez peu de critiques de particuliers ou de mentions d'événements historiques ${ }^{10}$.

\section{Dire par non-dire}

6 Si l'on ne trouve pas de critique de particuliers dans ces sotties, on y rencontre, en revanche, très souvent des clichés satiriques comme la corruption des moines, la simonie, l'avarice des usuriers et la fraude des boulangers. D'après la monographie sur la satire dans la sottie écrite par Heather Arden, la satire dans la sottie est, par sa nature, conservatrice à l'égard de la structure sociale ${ }^{11}$. Cependant, il est aussi vrai que certains auteurs de sottie représentent habilement la balance du pouvoir entre le chef 
des sots et ses sujets et, en le faisant, ils réussissent à dénoncer l'abus du pouvoir tant physique que rhétorique des gens qui dominent la hiérarchie sociale. À titre d'exemple, nous voulons nous pencher sur la scène du silence forcé. Dans des sotties ou des pièces assimilées à la sottie, se rencontre parfois la scène où un personnage impose le silence à un autre quand celui-ci continue de se plaindre : nous présentons un exemple tiré de la Sottie du Jeu du Prince des Sotz de Pierre Gringore :

LE PREMIER SOT

Trop [la Mère Sotte] a fait de mutinerie / Entre les princes et prelatz.

LA COMMUNE

Et j'en suis, par saincte Marie, / Tant plaine de melencolie / Que n'ay plus escuz ne ducas.

LE II

Tays toy, Commune, parle bas !12

7 En général, c'est un personnage plus ou moins « intelligent » qui impose le silence à un autre personnage niais comme «Chascun » ou «La Commune ». Par suite, cette scène du silence forcé nous invite à imaginer ce que ces personnages n'ont pas pu dire malgré eux et, de cette façon, le manque de liberté d'expression est mis au grand jour. Certes, il n'est pas toujours besoin de chercher la volonté satirique dans ces scènes du silence, mais il est aussi vrai que ces auteurs de sottie nous révèlent, inconsciemment ou non, le fait que la prise de la parole constitue un problème en soi dans un monde hiérarchisé. Par ailleurs, c'est surtout dans les pièces anonymes que le silence est imposé par un personnage dominant dans la hiérarchie des sots (dans la Sottie des sotz fourrés de malice $^{13}$, la Sottie de l'Astrologue ${ }^{14}$ et Pour le cry de la Bazoche $e^{15}$ ), alors que, dans les pièces signées, c'est un personnage moyennement placé dans la hiérarchie qui impose le silence au personnage niais (ce qui est le cas de la sottie de Pierre Gringore ou de la Satyre pour les habitans d'Auxerre de Roger de Collerye ${ }^{16}$ ). Malgré la fragilité de leur statut d'auteur par rapport aux écrivains officiels, ou justement parce qu'ils étaient anonymes, ces auteurs « mineurs » ont réussi, nous semble-t-il, à décrire le lien entre le silence forcé et le pouvoir dominant d'une façon moins compromettante.

8 Au moment où la satire est mise par écrit, peut-être surtout quand il s'agit des imprimés, elle doit être modifiée et transformée sous une forme plus abstraite et modérée ${ }^{17}$. Nous pourrions considérer la scène du silence forcé dans des sotties et des pièces assimilées à la sottie comme un des moyens de dépasser l'horizon poétique et politique qui contraignait les auteurs de sottie. L'interdiction de la critique des particuliers dans la littérature satirique correspond à l'exigence des autorités tant ecclésiastiques que royales. Comme nous l'avons constaté, les sotties conservées laissent peu de place à ce qui va à l'encontre de cette interdiction. Par la même raison, il me paraît encore plus difficile d'y trouver des traces de ce qui est un tabou dans la société de la fin du XV et du début du XVI ${ }^{\mathrm{e}}$ siècle, d'autant plus que le critère de tabou est, nous semble-t-il, difficilement précisé par l'écrit, contrairement au cas de l'interdiction. Le silence des sots niais est l'un des rares éléments qui nous permettent d'imaginer ce qui relève de l'interdit ou, éventuellement, du tabou. 


\section{NOTES}

1. Nous utiliserons l'appellation générique de « sottie " uniquement pour désigner les pièces qui se définissent telles quelles par leur titre. Mais nous devons mener une recherche codicologique plus poussée pour préciser notre choix, qui est fondé seulement sur les données décrites par les éditeurs précédents.

2. Cf. Pascal DEBAilly, "La satire lucilienne et la poétique du blâme ", in Poétiques de la Renaissance. Le modèle italien, le monde franco-bourguignon et leur héritage en France au XVIe siècle, sous la direction de Perrine GALAND-HALLYN et Fernand HALLYN, préface de Terence CAVE, Genève, Droz, 2001, p. 380 ; Olga TRTNIK-ROSSETTINI, Les influences anciennes et italiennes sur la satire en France au XVIe siècle, Firenze, Institut français de Florence, Edizioni Sansoni Antiquarito, pp. 7-72.

3. Josse BADE, Josse Bade, dit Badius (1460-1532). Préfaces de Josse Bade (1462-1535), humaniste, éditeurimprimeur et préfacier, traduction, introduction, notes et index par Maurice LEBEL, Louvain, Peeters, 1988, pp. 88-89 [Jodocus BADIUS, Prenotamenta ascensiana, in TERENTIUS, P. Terentii aphri comicorum elegantissimi Comedie [...], Lyon, Jacques Huguetan, 1511 (Bibliothèque Municipale de Lyon, fonds ancien, Rés. 160574, fol. viii, $\mathrm{R}^{\circ}$ )]. Nous avons modifié la traduction en fin de citation.

4. Cf. Jean BOUCHET, Epistres morales et familieres du Traverseur, Poitiers, Jacques Bouchet, 1545 (introduction par Jennifer BEARD, Mouton, Johnson Reprint Corporation, 1969), Epistres Morales, I, XIII, fol. xxxii, $\mathrm{V}^{\circ}$; L'INFORTUNÉ, L'Instructif de Seconde Rhétorique, in Le Jardin de Plaisance et Fleur de Rhétorique, fac-similé de l'édition de 1501, introduction et notes par E. DROZ et A. PIAGET, Paris, S.A.T.F., 1910, 2 vol., t. I, fol. c ii r ${ }^{\circ}$ b.

5. BOUCHET, éd. citée., Epistres Morales, I, XIII, fol. xxxii, $V^{\circ}$.

6. Cf. Michel ROUSSE, «Le pouvoir royal et le théâtre des farces ", in Le pouvoir monarchique et ses supports idéologiques aux XVI - XVII ${ }^{e}$ siècles, Paris, Publications de la Sorbonne nouvelle, 1990, pp. 185-197.

7. Nous pensons surtout à la Sottie du Jeu du Prince des Sotz de Pierre Gringore (Pierre GRINGORE, Le Jeu du Prince des Sotz et de Mère Sotte, édition critique par Alan HINDLEY, Paris, Champion, 2000, pp. 63-119). Cf. aussi Cynthia J. BROWN, « Political misrule and popular opinion : double talk and folly in Pierre Gringore's Jeu du Prince des Sotz ", in Le moyen français, 11, 1982, pp. 89-111. À propos $\mathrm{du}$ discours sur les particuliers dans les sotties, cf. aussi la réflexion d'Olga Anna Dull sur l'utilisation de l'allégorie dans les sotties (Olga Anna DULL, Folie et rhétorique dans la sottie, Genève, Droz, 1994, pp. 87-93).

8. Cf. Recueil général des sotties, éd. par Émile PICOT, Paris, S.A.T.F., 1902-1912, 3 vols, t. II, pp. 199-244.

9. Cf. ibid., t. I, pp. 195-231.

10. Cf. Le Recueil Trepperel : "Les Sotties », éd. par Eugénie DROZ, Paris, Droz, 1935 (Genève, Slatkine reprints, 1974); Le Recueil Trepperel : Fac-similé des trente-cinq pièces de l'original, avec une introduction par Eugénie DROZ, Genève, Slatkine reprints, [1966].

11. Cf. Heather ARDEN, Fool's Plays : A study of Satire in the "sottie ", Cambridge, Cambridge University Press, 1980. p. 74.

12. GRINGORE, éd. citée, p. 115, vv. 640-645.

13. Cf. Recueil Trepperel, éd. citée, pp. 83 et 84 , vv. 147-152.

14. Cf. Recueil général, éd. citée, t. I, p. 224, vv. 426-435.

15. Cf. ibid., t. III, pp. 115-147. Cette pièce n'est pas clairement intitulée comme « sottie ».

16. Cf. Sylvie LÉCUYER, Roger de Collerye. Un héritier de Villon, Paris, Champion, 1997, p. 177, vv. 267-273. Cette pièce n'est pas clairement intitulée comme « sottie». 
17. La tradition textuelle du Roman de Fauvel serait aussi très suggestive dans ce sens : cf. Joël BLANCHARD et Jean-Claude MÜHLETHALER, Écriture et pouvoir à l'aube des temps modernes, Paris, P.U.F., 2002, pp. 68 et 69. Cf. aussi l'étude sur la tradition textuelle de Maistre Pierre Pathelin par Darwin Smith (Maistre Pierre Pathelin. Le Miroir d'Orgueil, texte d'un recueil inédit du XV siècle (mss Paris, B.N.F. fr. 1707 et 15080), introduction, édition, traduction et notes par Darwin SMITH, Saint-Benoît-Du-Sault, Tarabuste, 2002, pp. 79-100).

INDEX

Mots-clés : satire, théâtre, tabou

Keywords : satire, theatre, taboo 